\title{
Relación entre las dimensiones de personalidad según el P-IPG y la calidad de vida laboral percibida
}

\author{
CARLOS ALBERTO GÓMEZ RADA* \\ Universidad Católica de Colombia, Bogotá, Colombia. \\ ORCID: https://orcid.org/0000-0003-4374-0343
}

How to quote this article: Gómez Rada, C.A. (2019). La relación entre las dimensiones de personalidad según el p-ipg y la calidad de vida laboral percibida. Acta Colombiana de Psicología, 22(1), 230-238. doi: http://www.dx.doi.org/10.14718/ ACP.2019.22.1.11

Resumen

\begin{abstract}
La calidad de vida laboral (CVL) y la personalidad constituyen dos de los tópicos de mayor interés en la investigación del comportamiento humano en la organización. La presente investigación tuvo como objetivo identificar si es posible predecir la CVL a partir de algunas dimensiones de personalidad. Para su desarrollo, se utilizó un diseño descriptivo correlacional con una muestra conformada por 145 trabajadores de cinco empresas del sector privado de la ciudad de Bogotá. La personalidad se evaluó por medio de la prueba PPG-IPG, de Gordon (1993), y para la calidad de vida se utilizó el PCVL, de Gómez-Rada (2010). Se analizaron los resultados por medio estadísticos descriptivos y de carácter correlacional entre las variables con los coeficientes producto momento de Pearson y los rangos de Spearman. Los resultados muestran que la valoración de la CVL se encuentra relacionada positivamente con el hecho de que el trabajador muestre una personalidad más positiva, en términos de mayor autoestima, cautela, originalidad, comprensión y vitalidad.

Palabras clave: calidad de vida laboral, personalidad, estudio correlacional, PPG-IPG, perfil de calidad de vida laboral.
\end{abstract}

\section{The relation between personality dimensions according to P-IPG and the perceived labor life quality}

\begin{abstract}
Labor life quality (CVL, for its Spanish acronym) and personality conform two of the most interesting topics in human behavior research within the organization. The aim of this research was to identify whether it was possible to predict CVL as of some personality dimensions. To do so a correlational descriptive design was used. The sampling was made up of 145 workers at 5 private sector companies in the city of Bogotá. Personality was evaluated through the Gordon PPG-IPG test, and for life quality, Gómez PCVL (2010) was used. The results were analyzed by means of descriptive statistics and the relation between variables was analyzed through Pearson moment-product coefficients and Spearman ranges. Results show that CVL valuation is positively related to the fact that the worker shows a more positive personality in terms of greater self-steem, caution, originality, understanding, and vitality.

Keywords: labor life quality, personality, correlational study, PPG-IPG, labor life quality profile.
\end{abstract}

\footnotetext{
Facultad de Psicología, avenida Caracas N. 46-40, Tel.: 5713277300, ext.: 5050. cagomez@ucatolica.edu.co Se agradece la participación de: Cindy Julieth Garcia Gómez, Diana Katherine Huérfano Güiza, Delvis Marinella Lengua Buelvas, Yuly Villamizar Montaño, Nora Camila Ospina, Natalia Rodríguez Delgadillo.
} 


\title{
Relação das dimensóes de personalidade segundo o P-IPG e a qualidade de vida profissional percebida
}

Resumo

\begin{abstract}
A qualidade de vida profissional (QVP) e a personalidade constituem dois dos tópicos de maior interesse na pesquisa do comportamento humano na organização. Esta pesquisa teve como objetivo identificar se é possível predizer a QVP a partir de algumas dimensões de personalidade. Para seu desenvolvimento, foi utilizado um desenho descritivo correlacional com uma amostra conformada por 145 trabalhadores de cinco empresas do setor privado da cidade de Bogotá, Colômbia. A personalidade foi avaliada por meio do teste PPG-IPG, de Gordon (1993), e para a qualidade de vida foi utilizado o PCVL, de Gómez-Rada e Ponce de León (2010). Foram analisados os resultados por meios estatísticos descritivos e de caráter correlacional entre as variáveis com os coeficientes produto momento de Pearson e as categorias de Spearman. Os resultados mostram que a avaliação da QVP se encontra relacionada positivamente com o fato de o trabalhador mostrar uma personalidade mais positiva, em termos de mais autoestima, cautela, originalidade, compreensão e vitalidade.

Palavras-chave: qualidade de vida profissional, personalidade, estudo correlacional, PPG-IPG, perfil de qualidade de vida profissional.
\end{abstract}

\section{Introducción}

Existen diversas aproximaciones al concepto de calidad de vida laboral (CVL) que coinciden en señalar que se trata de un constructo multidimensional que incluye diversas condiciones de la organización -como los aspectos físicos, el soporte institucional y los procesos de recursos humanos, como lo son la seguridad laboral, la integración al puesto de trabajo, las oportunidades de desarrollo laboral y la participación activa en la toma de decisiones y en el diseño de sus vidas de trabajo- que, además de generar un impacto en la motivación laboral de los trabajadores, protegen y promueven la satisfacción de los empleados en los ambientes de trabajo en tanto afectan su bienestar laboral (Chiavenato, 2005; González, Hidalgo, Salazar \& Preciado, 2009; Huerta, Pedraja, Contreras \& Almodóvar, 2011; Cañón \& Galeano, 2011).

Con respecto a la CVL, en la década de los 70, Walton desarrolló una propuesta en la Universidad de Harvard, adaptada posteriormente en Colombia por Gómez-Rada (2010), que plantea que este constructo está conformado por ocho factores: la compensación justa y adecuada, las condiciones de seguridad y salud en el trabajo, el empleo y desarrollo de la capacidad, las oportunidades de crecimiento continuo y seguridad, la integración social en la organización, la reglamentación, el trabajo y espacio total de vida, y la importancia social de la vida en el trabajo, y es este el modelo que se tendrá en cuenta para el desarrollo de la presente investigación.
Sin embargo, no existe solo un método para evaluar la CVL, por lo que en la literatura se ha optado por un pluralismo metodológico y por la combinación inexcusable de varios niveles y focos de análisis que han permitido valorar de forma sistemática e integrada todas las facetas que componen la vida en el trabajo (Gálvez, 2004), y que incluyen, por una parte, los métodos objetivos, que toman medidas cuantitativas del medio ambiente de trabajo, como la seguridad, la higiene ambiental, la iluminación y el nivel de ruido, entre otros; así como de la organización, entre los que figuran los horarios, el salario y la formación, etc.; y de los trabajadores, como el rendimiento, la carga física y la fatiga (Grueso \& Anton, 2011). Y, por otra parte, los métodos subjetivos, que evalúan la CVL percibida a partir de las opiniones y juicios que los trabajadores emiten acerca de su entorno de trabajo (Ruiz \& Ispizua, 1989, citado por Valles, 2014). Entre las técnicas de corte subjetivo que se basan en medidas cuantitativas y cualitativas se destacan la observación, la entrevista, la encuesta y macroencuesta, el grupo de discusión, el cuestionario y las escalas de satisfacción laboral (Royer, 2011).

Con respecto a lo anterior, en la literatura científica se puede observar que las encuestas son la estrategia metodológica que más se ha utilizado en el área, y dentro de este grupo se han desarrollado varios tipos, como el CVP-35, que mide la experiencia de bienestar derivada del equilibrio que percibe el individuo entre las demandas o cargas de trabajo desafiante y los recursos psicológicos, organizacionales y relacionales que dispone para afrontarlos (Quezada, Castro \& Cabezas, 2010); o el Perfil de Calidad 
de Vida Laboral (PCVL; Gómez-Rada, 2010), que evalúa las ocho dimensiones desarrolladas por Walton (1973), y que será la herramienta de trabajo en el presente estudio.

$\mathrm{Al}$ igual que con el concepto de calidad de vida en el trabajo, existen diversas aproximaciones para entender el constructo de personalidad que, según Atkinson y Hilgard (2003), convergen en un concepto que describe el crecimiento y desarrollo de todo el sistema psicológico de una persona; según Moreno, Garrosa y Gálvez (2005), está constituida por una serie de rasgos subyacentes que se infieren a partir de la conducta tanto manifiesta como de la experiencia privada de los individuos; y, según Furnham (2011), incluye las respuestas habituales de la persona ante situaciones cotidianas, lo que implica pautas uniformes de comportamiento que, aunque pueden cambiar, en general perduran a lo largo del tiempo, y se suelen manifestar en contextos específicos, como el emocional, el social, el moral, el sociocultural y el familiar (Ríos, 2008).

A partir de ahora, en el presente estudio se asumirá la personalidad desde la perspectiva de la teoría de rasgos, según la cual esta puede entenderse como el conjunto de rasgos que determinan la forma de comportarse de un individuo, y entre los cuales se encuentran la ascendencia, la responsabilidad, la estabilidad emocional, la sociabilidad, la cautela, la originalidad, las relaciones personales y el vigor (Rodríguez-Chavez \& Garibay-Bermúdez, 2014).

Ahora bien, con respecto a la evaluación de la personalidad, es importante destacar las técnicas proyectivas y las técnicas objetivas, estas últimas basadas en la teoría de rasgos (Boyle, Ortet \& Ibanez, 2001). Específicamente, dentro de la teoría de rasgos se pueden encontrar diversos instrumentos de frecuente aplicación en las organizaciones, como sucede con el 16PF, desarrollado por Cattel (s. f., citado por Papalia, Wendkos \& Duskin, 2010); el Inventario Psicológico de California (CPI), elaborado en la Universidad de Minnesota; el Eyseck Personality Inventory (EPI), realizado por Montaño, Palacios y Gantiva (2009); y la PPG-IPG, que evalúa, en particular, la teoría de rasgos (Rodríguez-Chavez \& Garibay-Bermúdez, 2014).

De los estudios que se han desarrollado en el contexto internacional que abordan la CVL y la personalidad, destaca el trabajo de Albanesi, Garelli y Casari (2003), en donde se llevó a cabo un estudio con el objetivo de indagar sobre los estilos de personalidad y la calidad de vida en estudiantes de la carrera de psicología, en el cual no fue posible detectar ninguna correlación positiva entre la calidad de vida y algún estilo de personalidad en particular, pero sí se pudo detectar múltiples correlaciones negativas, siendo la escala de bienestar social la que más relación obtuvo con los estilos de personalidad, sobre todo con los de carácter paranoide, esquizotípico y esquizoide.
De igual forma, sobresale el trabajo de Malekiha, Abedi y Baghban (2012), donde se examinó la relación entre el conflicto familia-trabajo y conflicto trabajo-familia, que es una de las dimensiones de la CVL, en comparación con los cinco grandes rasgos de la personalidad, donde los resultados mostraron que el neuroticismo y la conciencia estaban relacionados en ambas direcciones del conflicto, mientras que la extraversión no.

Por otra parte, estudios diferentes relacionan la personalidad no directamente con la CVL, pero sí con un constructo estrechamente relacionado: la satisfacción laboral. Al respecto, Heller, Lance, Brown y Watson (2009) realizaron dos estudios (uno transversal y uno longitudinal de más de un año de duración), cuyo objetivo era examinar la validez de la personalidad en el trabajo como factor predictor de la satisfacción laboral e identificar los efectos de la personalidad global sobre la satisfacción laboral. En el primer estudio los resultados mostraron que los individuos varían sistemáticamente en su personalidad según los roles y que los sujetos fueron significativamente más abiertos a la experiencia y menos extrovertidos en el trabajo, además de que la personalidad resultó ser un buen predictor de la satisfacción laboral; y en el segundo, se reclutó a los participantes a partir de la base de datos de exalumnos de una universidad canadiense, teniendo en cuenta que se seleccionaran egresados de una amplia variedad de organizaciones para reducir el impacto del contexto de la organización en las conclusiones, y se encontró que cada una de las dimensiones de la personalidad en el trabajo estaba mediada por la asociación entre sus correspondientes rasgos de personalidad y la satisfacción laboral.

Asimismo, Laca, Mayoral, Mejía y Yáñez (2010) dan cuenta de un estudio empírico intercultural que parte de los presupuestos de la psicología del bienestar subjetivo aplicados al ámbito laboral con un propósito general comparativo para estudiar la relación e influencia de factores sociodemográficos, de afectividad y de personalidad en el bienestar laboral de profesores no universitarios de España y México, e identificar el conjunto de factores que predicen mejor la satisfacción laboral intrínseca. Los resultados obtenidos en este trabajo indican la existencia de diferencias personales significativas en la percepción del bienestar laboral entre las dos culturas, y que la afectividad (positiva y negativa) predice la satisfacción laboral (alta y baja) para ambas muestras, siendo que para los profesores españoles los factores de afabilidad y apertura mental predicen un nivel de satisfacción alta, mientras que para los profesores mexicanos solo el factor de afabilidad resultó ser predictivo.

Además de lo anterior, la CVL se ha establecido como tópico de estudio en diferentes organizaciones, ocupa un lugar destacado en la gestión del talento humano y es uno de 
los factores primordiales dentro del entorno laboral (Barrios \& Paravic, 2006); mientras que la personalidad es una de las variables de comportamiento que más ha interesado a diversos autores y cuyos efectos se asocian a la motivación y al desempeño humano en la organización (Che, Beh, Uli \& Idris, 2006; Huang, Lee \& Chang, 2007). En este sentido, también se ha observado que existen factores individuales que pueden afectar y son afectados por la percepción de la calidad de vida y que constituyen un aspecto esencial para su consecución, entre estos, la personalidad, y esto se refuerza con las investigaciones de Albanesi et al. (2009), Heller et al. (2009), Huang et al. (2007) y Laca et al. (2010), ya que en sus trabajos señalan relaciones entre componentes de la personalidad y constructos asociados a la CVL.

No obstante, no se han realizado en sentido estricto estudios para entender la relación que se presenta entre estas dos variables y el efecto que origina el tipo de personalidad en la percepción de CVL, especialmente en el contexto colombiano, donde hay pocas investigaciones sobre este tópico y los intereses se han orientado más a medir las relaciones de la CVL con otras variables, como la disposición al cambio organizacional (García-Rubiano \& Aponte-Forero, 2016).

En consonancia con lo anterior, se denota la necesidad de una investigación donde se correlacione la calidad de vida laboral percibida y la personalidad, cuyos resultados permitan enriquecer el cuerpo incipiente de conocimientos sobre esta materia. Por ello, en la presente investigación se busca establecer las relaciones que se presentan entre la calidad de vida laboral y los rasgos de personalidad de un grupo de trabajadores de empresas de Bogotá.

\section{Método}

\section{Diseño}

Se utilizó un diseño de tipo descriptivo correlacional, debido a que se buscaba la cuantificación y medición de las variables para hallar el índice de correlación entre ellas y así determinar el grado de relación existente (Hernández, Fernández \& Baptista, 2016).

\section{Participantes}

La muestra del estudio estuvo conformada por $63 \mathrm{mu}-$ jeres $(43.4 \%)$ y 82 hombres (52.6\%); específicamente, se contó con cuatro grupos etarios $(18-25,26-35,36-45$ y 46-55 años) en los cuales había, respectivamente, 45 (31\%), 65 (44.8\%), $26(17.9 \%)$ y siete personas (4.8\%) (dos [1.4\%] eran datos perdidos); adicionalmente, 42 participantes $(29 \%)$ tenían menos de un año trabajando,
$73(50.3 \%)$ tenían entre 1 y 5 años, 23 (15.9\%) tenían entre 6 y 10 años, 6 (4.1\%) tenían más de 10 años de experiencia laboral, y dos eran datos perdidos (1.4\%).

Los participantes hacían parte de cinco empresas de la ciudad de Bogotá, Colombia, y la selección de las empresas se hizo por conveniencia y según su disponibilidad. El muestreo fue de tipo incidental, a partir de la disponibilidad (designada por las organizaciones) de los miembros para participar en este estudio. Se establecieron como criterios de inclusión y exclusión que estuvieran vinculados en el momento del estudio a una de estas organizaciones, que tuvieran una antigüedad mínima de tres meses, que contaran con un contrato laboral, y que trabajaran con una intensidad horaria semanal mínima de 48 horas.

\section{Instrumentos}

Se utilizó la prueba de personalidad PPG-IPG, diseñada por Gordon (1993, citado por Rodríguez-Chávez \& GaribayBermúdez, 2014), que evalúa ocho dimensiones o rasgos de personalidad a través de 38 ítems. Este instrumento presenta una confiablidad de .74 y .89 para el PPG y de .76 y .92 para el IPG, por medio del método de dos mitades, corregido por la fórmula de Spearman-Brown. La validez de constructo de la prueba se estableció mediante la correlación con otros instrumentos reconocidos que evalúan la personalidad, donde, por ejemplo, se obtuvieron correlaciones entre .37 y .66 para algunas dimensiones con el Inventario de Temperamento de Guilford-Zimmerman; entre .21 y .58 con el Inventario de Temperamento de Thurstone; y entre -.28 y .66 con el Adult Opinion Survey. Este instrumento está conformado por dos subpruebas, cada una de las cuales evalúa cuatro dimensiones de la personalidad: el PPG, que mide la ascendencia, la responsabilidad, la estabilidad emocional y la sociabilidad -al combinar las puntuaciones se ofrece una puntuación de autoestima-, y el IPG, que incluye la cautela, la originalidad, las relaciones personales y la vitalidad (Gordon, 1993, citado por Rodríguez-Chávez \& Garibay-Bermúdez, 2014). Para el presente estudio se obtuvo una puntuación de alfa de Cronbach de .86 .

Por otra parte, se evaluó la calidad vida laboral percibida por medio de la prueba PCVL, diseñada y validada por Gómez-Rada (2010), constituida por ocho dimensiones y 39 ítems que se responden en una escala tipo Likert de cuatro grados. Dichas dimensiones son: (a) compensación y beneficios, (b) condiciones del ambiente y del trabajo, (c) naturaleza de la tarea, (d) desarrollo y seguridad laboral, (e) democracia organizacional, (f) derechos fundamentales, (g) equilibrio laboral, e (h) impacto social. Este instrumento fue validado desde la perspectiva de la teoría de respuesta al ítem (TRI) con una muestra de 250 sujetos 
de tres empresas, donde los resultados muestran un nivel de ajuste alrededor de la media (1.00) y de los extremos (1.02), y un índice de confiabilidad integral de .97. Estos hallazgos se comprobaron en la aplicación actual, donde se obtuvo un nivel de ajuste alrededor de la media (1.03) y de los extremos (1.06), además de un índice de confiabilidad integral de .90. También, mediante el alfa de Cronbach se obtuvo una medida de consistencia interna de .92 .

\section{Procedimiento}

El desarrollo del presente estudio se realizó a través de las siguientes fases: (a) la conformación de la muestra de estudio, en la cual se contactó a las organizaciones y se les comunicó el propósito del estudio; (b) la recolección de datos, en la que se entregó a los trabajadores una copia del consentimiento informado donde se les explicaba el propósito de la investigación, la naturaleza de su participación, y se les garantizaba la confidencialidad de la información, además de que no habría repercusiones negativas para el ejercicio de sus cargos (Resolución 8430 de 1993 del Ministerio de Salud; Código Deontológico de la Psicología establecido para Colombia en la ley 1090 de 2006, capítulo II -de la competencia profesional y la relación con otros profesionales, articulo 18-, Capítulo IV -de la investigación y docencia- y Capítulo V - de la obtención y uso de la información-). Los instrumentos se aplicaron en grupos de 10 personas, en un salón amplio y cómodo, con buena visibilidad e iluminación, dotado de sillas tipo estudiante y debidamente aislado de interferencias; (c) la elaboración de una base de datos en Excel para proceder al análisis de los datos obtenidos mediante el Software SPSS; y (d) la discusión de los resultados a la luz de los desarrollos conceptuales y empíricos precedentes.

\section{Análisis de datos}

A continuación se presenta el análisis descriptivo de los datos obtenidos para cada una de las dimensiones tanto de la personalidad como de la CVLP, se establece la normalidad de la muestra por medio de la prueba Kolmogorov-Smirnov y se da cuenta de las medidas de correlación pertinentes (Pearson o Spearman) para establecer la relación entre las variables de estudio.

\section{Consideraciones éticas}

Se tuvieron en cuenta los lineamientos de la resolución 8430 de 1993 del Ministerio de Salud, por la cual se establecen las normas científicas, técnicas y administrativas para la investigación en salud, sobre todo en lo concerniente al artículo 6 del capítulo II. También se tuvo en cuenta el código deontológico de psicología establecido para Colombia en la ley 1090 de 2006, principalmente en los lineamientos de el capítulo II -de la competencia profesional y de la relación con otros profesionales, articulo 18-, el capítulo IV -de la investigación y docencia- y el capítulo $\mathrm{V}$-de la obtención y uso de la información-. Finalmente, se tuvo en cuenta la ley 1581 de 2012, por la cual se dictan las disposiciones generales para la protección de datos personales.

\section{Resultados}

Para el análisis de los resultados, se inició con una descripción de las puntuaciones obtenidas en las dos variables de estudio, utilizando medidas de tendencia central y dispersión; a continuación, se procedió a establecer la normalidad de los datos con el fin de establecer los estadísticos pertinentes; $y$, por último, se hicieron las correlaciones correspondientes entre las variables de estudio y sus distintas dimensiones.

En primer lugar, en la Tabla 1 se indican las puntuaciones brutas, percentilares, porcentajes y la desviación obtenidas para las dimensiones de personalidad evaluadas por el P-IPG y para las dimensiones de CVL, evaluadas por el PCVL. Para el caso de la personalidad, se puede observar que las puntuaciones son relativamente moderadas para cada uno de los rasgos, y dentro del rango "moderado", la estabilidad emocional y la vitalidad presentaron las puntuaciones más altas, mientras que la cautela y la autoestima registraron las puntuaciones más bajas. Estos resultados señalan que en la muestra de estudio la ausencia de hipersensibilidad, ansiedad, preocupaciones y tensión nerviosa, además de la presencia de vigor y energía, se encuentran en un grado de normalidad. Por su parte, los rasgos relacionados con el cuidado a la hora de tomar una decisión, el agrado por llevar cabo una tarea difícil y la curiosidad intelectual, son características menos predominantes en los participantes. Los datos de desviación típica indican una muestra relativamente uniforme.

Además de esto, para la variable calidad de vida laboral las dimensiones que presentaron una puntuación más alta fueron las condiciones del ambiente y del trabajo e impacto social, mientras que las que obtuvieron una puntuación más baja fueron el desarrollo y la seguridad en el empleo, seguidas de la compensación y los beneficios, aunque su comportamiento es positivo. Esto indica que la percepción más positiva está relacionada con la confortabilidad y seguridad de las instalaciones, del equipamiento y de los horarios de los lugares de trabajo.

También, se presenta una percepción positiva con respecto a que las organizaciones funcionan dentro de parámetros de legalidad, responsabilidad social y cuidado del medio ambiente. Sin embargo, se percibe en forma menos 
positiva las posibilidades de desarrollo en la organización, la estabilidad en el empleo y la distribución de beneficios. La desviación típica es relativamente uniforme para cada una de las dimensiones de la CVL.

Tabla 1.

Puntuaciones descriptivas para las medidas de personalidad y CVL

\begin{tabular}{lccc}
\hline \multicolumn{1}{c}{ Dimensión } & $\begin{array}{c}\text { Puntuación } \\
\text { bruta }(\overline{\mathrm{x}})\end{array}$ & Percentiles & $\begin{array}{c}\text { Desviación } \\
\text { típica }\end{array}$ \\
\hline ASC & \multicolumn{2}{c}{ Prueba PPG-IPG } & \\
RESP & 21.7 & 43 & 4.9 \\
EST EMO & $25_{\mathrm{me}}$ & 41 & 5.5 \\
SOC & 23.2 & 46 & 5.1 \\
CAU & 20.4 & 36 & 4.05 \\
ORIG & 23.1 & 37 & 4.5 \\
COM & 23.7 & 40 & 4.2 \\
VIT & 23 & 39 & 4.8 \\
AUT & 24.5 & 45 & 5.5 \\
& $94_{\text {me }}$ & 36 & 15.1 \\
CB & Prueba PCVL & \\
CAT & 14.3 & $71.5 \%$ & 3.0 \\
NT & 19.7 & $82.1 \%$ & 2.8 \\
DSL & 19 & $79.2 \%$ & 2.8 \\
DO & $9_{\text {me }}$ & $70 \%$ & 2.3 \\
DF & 14.7 & $73.5 \%$ & 2.8 \\
EL & $13_{\text {me }}$ & $79.4 \%$ & 2.25 \\
IS & $12_{\text {me }}$ & $74.4 \%$ & 2.6 \\
\hline
\end{tabular}

Nota. ASC $=$ ascendencia $;$ RESP $=$ responsabilidad; EST EMO = estabilidad emocional; $\mathrm{SOC}=$ sociabilidad $; \mathrm{CAU}=$ cautela; ORIG = originalidad; $\mathrm{COM}=$ comprensión; $\mathrm{VIT}=$ vitalidad; $\mathrm{AUT}=$ autoestima; $\mathrm{CB}=$ compensación y beneficios; $\mathrm{CAT}=$ condiciones del ambiente y del trabajo; $\mathrm{NT}=$ naturaleza de la tarea; $\mathrm{DSL}=$ desarrollo y seguridad laboral; $\mathrm{DO}=$ democracia organizacional; $\mathrm{DF}=$ derechos fundamentales; $\mathrm{EL}=$ estabilidad laboral; IS = impacto social.

En segundo lugar, se realizó la prueba de KolmogorovSmirnov para establecer si los datos analizados en la muestra de estudio corresponden a una población con distribución normal. Al observar la Tabla 2 se puede apreciar que los datos obtenidos para las dimensiones tanto la prueba PPGIPG como para la prueba PCVL señalan que los datos de ocho de las dimensiones de personalidad y cinco de las dimensiones de la CVL corresponden a una distribución normal, con niveles de significación que varían entre .004 y .402. En contraste, las dimensiones de responsabilidad y autoestima del P-IPG, así como las dimensiones de desarrollo y seguridad laboral, derechos fundamentales y equilibrio laboral (del PCVL), no presentan una distribución normal.
Tabla 2.

Resultados prueba Kolmogorov-Smirnov para la muestra

\begin{tabular}{lcc}
\hline \multicolumn{1}{c}{ Dimensión } & Resultado & Significación \\
\hline Ascendencia & DN & .265 \\
Responsabilidad & & .044 \\
Estabilidad emocional & DN & .182 \\
Sociabilidad & DN & .112 \\
Cautela & DN & .078 \\
Originalidad & DN & .402 \\
Comprensión & DN & .306 \\
Vitalidad & DN & .218 \\
Autoestima & & .022 \\
Compensación y beneficios & DN & .182 \\
Condiciones del ambiente y del & DN & .162 \\
trabajo & & .229 \\
Naturaleza de la tarea & DN & .004 \\
Desarrollo y seguridad laboral & & .117 \\
Democracia organizacional & DN & .035 \\
Derechos fundamentales & & .015 \\
Equilibrio laboral & & .159 \\
Impacto social & & DN
\end{tabular}

Nota. $\mathrm{DN}=$ distribución normal.

En tercer lugar, se realizó una correlación entre las dimensiones de las variables personalidad y la CVL, para lo cual se utilizaron los coeficientes de correlación producto momento de Pearson para las que presentaban distribución normal, y el coeficiente de correlación de rangos de Spearman para las que no presentaban distribución normal (véase Tabla 3).

Como se puede observar, las magnitudes de las correlaciones entre las dimensiones de la personalidad y la CVL fueron moderadas y en la mayoría de los casos su dirección fue positiva, aunque en algunos fue negativa. Por ejemplo, la ascendencia presenta una correlación positiva con las condiciones del ambiente y del trabajo, la naturaleza de la tarea y el impacto social, siendo la asociación mayor con esta última; al igual que la responsabilidad, que muestra una correlación positiva con las condiciones del ambiente y del trabajo, la naturaleza de la tarea y el impacto social, siendo la asociación mayor con esta última, con un nivel de significación de .01 , pero una correlación negativa con la compensación y beneficios, y el desarrollo y seguridad laboral, ambas a un nivel de significación de .01.

Asimismo, la estabilidad emocional mantuvo una correlación positiva con las condiciones del ambiente y del trabajo, la naturaleza de la tarea y el impacto social, siendo con esta última la asociación mayor, las tres a un nivel de significación de .01. También, la sociabilidad presentó una correlación positiva con las condiciones del ambiente y del trabajo y el impacto social, ambas con un nivel de 
Tabla 3.

Correlaciones entre dimensiones de la personalidad y la CVL

\begin{tabular}{lcccccccc}
\hline \multicolumn{1}{c}{$\begin{array}{c}\text { Dimensión } \\
\text { Personalidad/CVL }\end{array}$} & $\mathrm{CB}$ & $\mathrm{CAT}$ & $\mathrm{NT}$ & $\mathrm{DSL}$ & $\mathrm{DO}$ & $\mathrm{DF}$ & $\mathrm{EL}$ & $\mathrm{IS}$ \\
\hline ASC & -.094 & $.172^{*}$ & $.167^{*}$ & $-.075 \mathrm{~s}$ & .012 & $.084 \mathrm{~s}$ & $-.011 \mathrm{~s}$ & $.290^{*}$ \\
RESP & $-.238 \mathrm{~s}^{* *}$ & $.220 \mathrm{~s}^{* *}$ & $.203 \mathrm{~s}^{*}$ & $-.211 \mathrm{~s}^{*}$ & $-.071 \mathrm{~s}$ & $.042 \mathrm{~s}$ & $.045 \mathrm{~s}$ & $.316 \mathrm{~s}^{* *}$ \\
EST EMO & -.057 & $.270^{* *}$ & $.301^{* *}$ & $-.080 \mathrm{~s}$ & .064 & $.164 \mathrm{~s}$ & $.139 \mathrm{~s}$ & $.392^{* *}$ \\
SOC & .030 & $.179^{*}$ & .161 & $-.009 \mathrm{~s}$ & .021 & $.062 \mathrm{~s}$ & $.080 \mathrm{~s}$ & $.189^{*}$ \\
CAU & $-.168^{*}$ & .046 & .126 & $-.198 \mathrm{~s}^{*}$ & -.048 & $.012 \mathrm{~s}$ & $-.039 \mathrm{~s}$ & .132 \\
ORIG & .036 & .278 & $.300^{* *}$ & $.065 \mathrm{~s}$ & .070 & $.194 \mathrm{~s} *$ & $.141 \mathrm{~s}$ & $.385^{* *}$ \\
COM & -.014 & $.231^{* *}$ & $.177^{*}$ & $.092 \mathrm{~s}$ & .107 & $.247 \mathrm{~s} *$ & $.134 \mathrm{~s}$ & $.268^{* *}$ \\
VIT & $-.210^{*}$ & $.171^{*}$ & $.209^{*}$ & $-.188 \mathrm{~s} *$ & -.117 & $.028 \mathrm{~s}$ & $.029 \mathrm{~s}$ & $.278^{* *}$ \\
AUT & $-.128 \mathrm{~s}$ & $.254 \mathrm{~s}^{* *}$ & $.267 \mathrm{~s}^{* *}$ & $-.131 \mathrm{~s}$ & $.024 \mathrm{~s}$ & $.107 \mathrm{~s}$ & $.070 \mathrm{~s}$ & $.338 \mathrm{~s}^{* *}$ \\
\hline
\end{tabular}

Nota. ASC $=$ ascendencia; RESP $=$ responsabilidad; EST EMO $=$ estabilidad emocional; SOC $=$ sociabilidad CAU $=$ cautela; $\mathrm{ORIG}=$ originalidad; $\mathrm{COM}=$ comprensión; $\mathrm{VIT}=$ vitalidad; $\mathrm{AUT}=$ autoestima; $\mathrm{CB}=$ compensación y beneficios; $\mathrm{CAT}=$ condiciones del ambiente y del trabajo; $\mathrm{NT}=$ naturaleza de la tarea; $\mathrm{DSL}=$ desarrollo y seguridad laboral; $\mathrm{DO}=$ democracia organizacional; $\mathrm{DF}=$ derechos fundamentales; $\mathrm{EL}=$ estabilidad laboral; $\mathrm{IS}=$ impacto social.

significación de .05. Mientras que la autoestima, mostró una correlación positiva con las condiciones del ambiente y del trabajo, la naturaleza de la tarea y el impacto social, siendo con esta última la asociación mayor, y un nivel de significación de .01 para las tres.

Por otra parte, la cautela mostró una correlación negativa con la compensación y beneficios y con el desarrollo y seguridad laboral, con la primera a un nivel de significación de .05 y con la segunda de .01. Mientras que la originalidad sostuvo una correlación positiva con la naturaleza de la tarea, los derechos fundamentales, el equilibrio laboral y el impacto social, las cuatro con un nivel de significación de .01. También, la comprensión tuvo una correlación positiva con las condiciones del ambiente y del trabajo, la naturaleza de la tarea, los derechos fundamentales y el impacto social, la segunda a un nivel de significación de .05 y las otras tres a .01 .

Finalmente, la vitalidad presentó una correlación positiva con las condiciones del ambiente y del trabajo, la naturaleza de la tarea y el impacto social, siendo mayor con esta última la asociación mayor, a un nivel de significación de .01 , mientras con las primeras fue de .05; y una correlación negativa con la compensación y beneficios con el desarrollo y seguridad laboral, la primera a un nivel de significación de .05, y la última, a .01.

\section{Discusión}

Los resultados hallados en este estudio confirman la idea de que se presentan relaciones entre la personalidad y la CVL. Sin embargo, estas asociaciones no se dan entre los constructos generales, sino entre ciertos rasgos de la personalidad y ciertas dimensiones de la calidad de vida laboral. Algunas de estas relaciones muestran una asociación positiva en el mismo sentido en que lo sugieren los estudios de Huang et al. (2007), Heller et al. (2009) y Malekiha et al. (2012); mientras que otras muestran una correlación negativa que coincide con los hallazgos de Albanesi et al. (2003). Sin embargo, es importante aclarar que los estudios anteriores no evaluaron la CVL en sentido estricto ni tuvieron en cuenta las mismas dimensiones del presente estudio.

Con respecto a las asociaciones positivas encontradas, es decir, entre las dimensiones de personalidad de ascendencia, responsabilidad, estabilidad emocional, sociabilidad y autoestima, y las condiciones del ambiente del trabajo, la naturaleza de la tarea y el impacto social, estas sugieren que en la medida en que la persona tiene una apreciación más positiva de sí misma, ello incide en la valoración que hace de las condiciones físicas y de equipamiento, así como del horario, la posibilidad de utilizar sus capacidades laborales y la autonomía para hacerlo, e incluso la manera como la organización asume su responsabilidad social. Ello puede explicarse, por una parte, en la medida que una buena autoestima contribuye a mejorar la motivación de las personas en el contexto laboral (Che et al., 2006), y, por otra, porque genera comportamientos proactivos para aumentar su propio bienestar (Huang et al., 2007).

En este mismo sentido, las dimensiones de personalidad referentes a la originalidad, la comprensión y la vitalidad mostraron una correlación positiva con las dimensiones del CVL que hacen referencia a las condiciones del ambiente y del trabajo, la naturaleza de la tarea y el impacto social 
(en el caso de originalidad, solo con las dos últimas). Esto podría indicar que, en general, una persona que presenta una estructura de personalidad más positiva, en términos de estar más orientada hacia retos intelectuales, tareas difíciles, confianza y tolerancia hacia los demás (con energía y vitalidad), y a hacer valoraciones más positivas de las dimensiones mencionadas de la CVL. Esto se encuentra en consonancia con lo encontrado por Laca et al. (2010), debido a que estos autores establecieron que las dimensiones de personalidad de afabilidad y apertura mental predicen un nivel alto de satisfacción.

En contraste, las dimensiones de personalidad referentes a la responsabilidad, la cautela y la vitalidad mostraron correlaciones negativas con las dimensiones de compensación y beneficios, y desarrollo y seguridad laboral de la CVL. Este aspecto no aparece en ninguno de los estudios revisados con antelación, dentro de los cuales se presentan relaciones negativas solo cuando hay alguna psicopatología, pero no en personas sanas (Albanesi et al., 2003). Una posible explicación para este hallazgo podría estar relacionada con que las expectativas que tienen las personas más constantes y perseverantes en las tareas propuestas (así no sean de su agrado), más cuidadosas a la hora de tomar decisiones, que trabajan con mayor rapidez y que hacen más cosas que los demás, es decir, con más responsabilidad, cautela y vitalidad, pueden tener mayores salarios y contar con otros beneficios como contraprestación a su contribución de la organización, como lo son las posibilidades de crecimiento en la organización y la estabilidad en el empleo, es decir, lo que tiene que ver con las dimensiones de compensación y beneficios, y de desarrollo y seguridad laboral. De este modo, al puntuar más alto en esas dimensiones de la personalidad, la percepción subsecuente de dichas dimensiones de CVL tiende a tener una valencia negativa. Estos resultados confirman los hallazgos realizados por Gómez y Ponce de León (2010) en el sentido de que el salario es la variable más relevante para explicar la percepción de la CVL.

Por otra parte, las dimensiones de personalidad referentes a la originalidad y la comprensión fueron las únicas que mostraron una correlación positiva con la dimensión de derechos fundamentales de la escala de CVL. Esto implica que el gusto por las tareas difíciles, la curiosidad intelectual, la fe en los demás, la tolerancia y paciencia hacia estos tendería a generar una valoración más positiva con respecto a la forma como es tratado por los demás y a observar una mayor libertad de palabra, un trato más equitativo, procesos disciplinarios justos, respeto por su privacidad y ausencia de violencia laboral (psicológica o física). Este aspecto se puede explicar, en parte, debido a que la tolerancia hacia los demás constituye un componente clave de la inteligencia emocional. En este sentido, de acuerdo con un estudio realizado por Ziauddini y Naroei (2013), se ha encontrado una relación positiva entre la inteligencia emocional y la CVL, posiblemente debido a que las personas que tienen mayor inteligencia emocional pueden vivir cómodamente con los demás, sentirse útiles y aceptados por los demás y, finalmente, tener la oportunidad de aumentar sus habilidades y conocimientos.

Adicionalmente, la originalidad también presentó una asociación positiva con el equilibrio laboral, lo que supone que el gusto por las tareas difíciles y la curiosidad intelectual genera valoraciones más positivas con respecto a que los roles que desempeñan en su trabajo no afectan negativamente el desarrollo en otras dimensiones de su vida personal, cómo la familia, la recreación, la vida comunitaria o el descanso. Esto es coherente con lo planteado en el modelo de Hackman y Oldham (1975, citados en Furnham, 2011), en el sentido que un mayor grado de identidad con el cargo se traduce en una mayor satisfacción laboral, y ello, a su vez, produciría una percepción más favorable de la CVL.

Por último, es importante considerar que los hallazgos aquí presentados permiten pensar que la personalidad juega un papel importante en la valoración de la CVL; no obstante, para futuros estudios debe considerarse el hecho de trabajar con muestras más extensas y con mayor diversidad poblacional, lo que permitiría hacer una mayor generalización de los resultados. Adicionalmente, desde el punto de vista metodológico valdría la pena utilizar aproximaciones más robustas por medio de técnicas de análisis multivariado para evaluar cómo se puede explicar la percepción de la CVL a partir de la personalidad. Ello implicaría preguntarse en qué grado la personalidad explica la percepción de la CVL y cuál es su peso diferencial con respecto a las variables del contexto organizacional.

\section{Referencias}

Albanesi, S., Garelli, V., \& Casari, L. (2003). Estilos de Personalidad y Calidad de Vida en Estudiantes de Psicología. Fundamentos en Humanidades, 19(1), 139-156. doi: 10.13140/RG.2.1.1761.5520

Atkinson, R., \& Hilgard, E. (2003). Introducción a la Psicología (14. ${ }^{\mathrm{a}}$ ed.). Madrid: Ed. Thomson.

Barrios, S., \& Paravic, T. (2006). Health promotion and a healthy workplace. Revista Latino-Americana de Enfermagem, 14(1), 136-141. doi:10.1377/hlthaff.2009.0626

Boyle, G., Ortet, G., \& Ibanez, M. (2001). Evaluación de la personalidad y la inteligencia: Una perspectiva cattelliana. Universitas Tarraconensis Revista de Psicología, 23(1-2), 7392. Recuperado de www.tdx.cat/bitstream/10803/10514/2/ escriva.pdf.txt 
Cañón, S., \& Galeano, G. (2011). Factores laborales psicosociales y calidad de vida laboral de los trabajadores de la salud de ASSBASALUD E.S.E Manizales (Colombia). Archivos de Medicina, 11(2), 114-216. Recuperado de www. redalyc.org/pdf/2738/273821489004.pdf

Che, R., Beh, L., Uli, J., \& Idris, K. (2006). Quality of Work Life: Implications of Career Dimensions. Journal of Social Sciences, 28(2), 61-67. Recuperado de https://www.research gate.net/.../26445222_Quality_Of_Work_Lif.

Chiavenato, I. (2005). Calidad de vida laboral. En Gestión del talento humano. Primera Edición. Colombia: McGrawHill.

Furnham, A. (2011). El comportamiento del individuo en las organizaciones. En A. Furnham, Psicología de las Organizaciones. Mexico: Alfaomega.

Gálvez, N. (2004). La conciliación de la vida laboral y familiar como proyecto de calidad de vida desde la igualdad. BOE, 5, 91-107. doi: http://dx.doi.org/10.5565/rev/pa pers/v83n0.1136

García-Rubiano, M., \& Aponte-Forero, C. (2016). Calidad de Vida Laboral y la disposición al cambio organizacional en funcionarios de empresas de la ciudad de Bogotá, Colombia. Revista Acta Colombiana de Psicología, 19(1), 79-90. doi: http://www.dx.doi.org/10.14718/ACP.2016.19.1.5

Gómez-Rada, C. A. (2010). Diseño, construcción y validación de un instrumento que evalúa calidad de vida laboral percibida en organizaciones Colombianas, desde la teoría de respuesta al ítem. Revista Psychologia. Avances de la Disciplina, 4(1), 113-124. Recuperado de http://www.redalyc. org articulo. $.0 \mathrm{a} \cdot \mathrm{id}=297224086010$

Gómez-Rada, C. A., \& Ponce de León, E. (2010). Modelo integrativo de la calidad de vida laboral percibida a partir de la inseguridad laboral, la jornada laboral y el salario, para hombres y mujeres en diferentes rangos de edad. Revista Psychologia. Avances de la Disciplina, 4(2), 113129. Recuperado de http://www.redalyc.org/articulo. oa? $\mathrm{id}=297224090008$

González, R., Hidalgo, G., Salazar, J., \& Preciado M. (2009). Instrumento para medir la Calidad de Vida en el Trabajo CVT-GOHISALO. Manual para su aplicación e interpretación (1. ed.). Guadalajara: Ediciones de la Noche.

Grueso, M., \& Anton, M. (2011). Prácticas de conciliación entre la vida personal y laboral: Comparación entre muestras procedentes de España y Colombia. Investigación y Desarrollo, 19(1), 42-63. Recuperado de www.esai.edu.ec/staff/ merlin-grueso/

Heller, D., Lance, F., Brown, D., \& Watson D. (2009). The Influence of Work Personality on Job Satisfaction: Incremental Validity and Mediation Effects. Journal of Personality, 77(4), 1051-1084. doi: https://doi.org/10.1111/j.14676494.2009.00574.x

Hernández, R., Fernández, C., \& Baptista, P. (2016). Metodología de la investigación (6. ${ }^{\mathrm{a}}$ ed.). México: Mc Graw Hill Interamericana.
Huang, C., Lee, L., \& Chang, M. (2007). The influences of personality and motivation on exercise participation and quality of life. Social Behavior And Personality, 35(9), 1189 -1210. Recuperado de https://www.sbp-journal.com/index. $\mathrm{php} / \mathrm{sbp} /$ article/view/1649

Huerta, P., Pedraja, L., Contreras, S., \& Almodóvar, P. (2011). Calidad de vida laboral y su influencia sobre los resultados empresariales. Revista de Ciencias Sociales, 17(4), 658-67. Recuperado de http://www.redalyc.org/articulo. oa? $\mathrm{id}=28022784008$

Laca, F., Mayoral, E., Mejía, J., \& Yáñez, C. (2010). Factores afectivos y de personalidad como antecedentes del bienestar laboral en profesores españoles y mexicanos. Pensamiento Psicológico, 9(16), 11-26. Recuperado de https://books. google.com.co/books?isbn=9401790353

Malekiha, M., Abedi, M., \& Baghban, I. (2012). Work-Family conflict and personality. Interdisciplinary Journal of Contemporary Research in Business, 3(10), 144-152. Recuperado de https://pdfs.semanticscholar.org/.../710e5ae8a96bd 49801cd45a9a

Montaño, M., Palacios, J., \& Gantiva, C. (2009). Teorías de la personalidad. Un análisis histórico del concepto y su medición. Psychologia. Avances de la disciplina, 3(2), 81-107. Recuperado de www.redalyc.org/articulo. oa? id $=297225531007$

Moreno, B., Garrosa, E., \& Gálvez, M. (2005). Personalidad Positiva y Salud. En Psicología de la salud. Temas actuales de investigación en Latinoamérica. Bogotá: ALAPSA.

Papalia, D., Wendkos, S., \& Duskin, R. (2010). Desarrollo humano (11. ${ }^{\mathrm{a}}$ ed.). México: Mc Graw Hill Interamericana.

Quezada, F., Castro, A., \& Cabezas, F. (2010). Diagnóstico de la Calidad de Vida Laboral percibida por los trabajadores de cuatro servicios clínicos del complejo asistencial "Dr. Víctor Ríos Ruiz" de Los ángeles (CAVRR). Horizontes Empresariales, 9(1), 55-68. Recuperado de revistas.ubiobio.cl/ index.php/HHEE/article/view/2037

Ríos, J. (2008). Personalidad, Madurez Humana y Contexto Familiar. Madrid: Editorial C.C.S.

Rodríguez-Chávez, C., \& Garibay-Bermúdez, J. R. (2014). Análisis de rasgos de personalidad en estudiantes de licenciatura en administración de una IES. Ciudad de Panamá: Asamblea general de la ALAFEC.

Royer, C. (2011). El mejoramiento de la Calidad de Vida Laboral como estrategia para vigorizar la capacidad de gestión municipal. DAAPGE, 16, 157-162. Recuperado de www. redalyc.org/pdf/3375/337530220005.pdf

Valles, M. (2014). Entrevistas cualitativas (Vol. 32). Madrid: CIS-Centro de Investigaciones Sociológicas.

Ziauddini, M., \& Naroei, H. (2013). Examined the Relationship between Emotional Intelligence and Quality of Working Life City Executive Personnel Khash. International Journal of Academic Research in Business and Social Sciences, 3(11), 337-345. 